\title{
Assessment of Graft Maturity After Anterior Cruciate Ligament Reconstruction Using Autografts: A Systematic Review of Biopsy and Magnetic Resonance Imaging studies
}

\author{
Bart van Groningen, M.D., M. C. (Marieke) van der Steen, Ph.D., Daan M. Janssen, B.Sc., \\ Lodewijk W. van Rhijn, M.D., Ph.D., Antoine N. van der Linden, M.D., and \\ Rob P. A. Janssen, M.D., Ph.D.
}

\begin{abstract}
Purpose: The purpose of this investigation was to evaluate systematically the literature concerning biopsy, MRI signal to noise quotient (SNQ) and clinical outcomes in graft-maturity assessment after autograft anterior cruciate ligament reconstruction (ACLR) and their possible relationships. Methods: The systematic review was reported and conducted according to the PRISMA (Preferred Reporting Items for Systematic reviews and Meta-Analyses) guidelines. Studies through May 2019 evaluating methods of intra-articular ACL autograft maturity assessment were considered for inclusion. Eligible methods were histologic studies of biopsy specimens and conventional MRI studies reporting serial SNQ and/ or correlation with clinical parameters. Results: Ten biopsy studies and 13 imaging studies, with a total of 706 patients, met the inclusion criteria. Biopsy studies show that graft remodeling undergoes an early healing phase, a phase of remodeling or proliferation and a ligamentization phase as an ongoing process even 1 year after surgery. Imaging studies showed an initial increase in SNQ, peaking at approximately 6 months, followed by a gradual decrease over time. There is no evident correlation between graft SNQ and knee stability outcome scores at the short- and long-term follow-up after ACLR. Conclusions: The remodeling of the graft is an ongoing process even 1 year after ACLR, based on human biopsy studies. MRI SNQ peaked at approximately 6 months, followed by a gradual decrease over time. Heterogeneity of the MRI methods and technical restrictions used in the current literature limit prediction of graft maturity and clinical and functional outcome measures by means of MRI graft SNQ after ACLR. Level of evidence: Level IV, systematic review, including level III and IV studies.
\end{abstract}

From the Orthopaedic Center Máxima, Máxima Medical Center, Eindhoven, the Netherlands (B.v.G., M.C.v.d.S., D.M.J., R.P.A.J.); Department of Orthopaedic Surgery, Catharina Hospital Eindhoven, Eindhoven, the Netherlands (M.C.v.d.S.); Department of Orthopaedic Surgery, Maastricht University Medical Center, Maastricht, the Netherlands (L.W.v.R.); Department of Radiology, Máxima Medical Center, Eindhoven, the Netherlands (A.N.v.d.L.); Fontys University of Applied Sciences, Eindhoven, the Netherlands (R.P.A.J.); Orthopaedic Biomechanics, Department of Biomedical Engineering, Eindhoven University of Technology, Eindhoven, the Netherlands (R.P.A.J.); and Maastricht University, Maastricht, the Netherlands (D.M.J.).

The authors report no conflicts of interest in the authorship and publication of this article. Full ICMJE author disclosure forms are available for this article online, as supplementary material.

Received October 8, 2019; accepted February 18, 2020

Address correspondence to Bart van Groningen, M.D., Orthopaedic Center Máxima, Máxima Medical Center, PO Box 90052, 5600 PD Eindhoven, the Netherlands.E-mail:b.vangroningen@mmc.nl

(C) 2020 THE AUTHORS. Published by Elsevier Inc. on behalf of the Arthroscopy Association of North America. This is an open access article under the CC BY-NC-ND license (http://creativecommons.org/licenses/by-nc-nd/4.0/).

2666-061X/191219

https://doi.org/10.1016/j.asmr.2020.02.008 nterior cruciate ligament reconstruction (ACLR) A has become a common procedure in the field of orthopedic sports medicine to restore stability in the knee and promote return to sport after rupture of the anterior cruciate ligament. ${ }^{\text {}}$ After surgery, the graft tissue changes from tendinous to ligamentousappearing in the new intra-articular environment, a process also called remodeling. ${ }^{2}$ Animal and human in vitro and in vivo research has demonstrated 3 characteristic stages of graft healing after ACLR: an early healing phase, followed by a phase of proliferation and, finally, a maturation or ligamentization phase. ${ }^{3-5}$ During these phases of healing, changes in cellularity, vascularity and extracellular matrix transform graft characteristics into properties of the intact ACL. ${ }^{6,7}$ However, full restoration of the biologic properties is not achieved. ${ }^{8,9}$

Optimal timing for return to sport after ACLR remains an on-going debate. ${ }^{10,11}$ To prevent graft reinjury, the 
safe return to sport is of great concern for both patients and physicians. ${ }^{12,13}$ Knee stability and patient-reported outcome measures (PROMs) have traditionally been used to evaluate the success of ACLR and timing of return to sport. ${ }^{10,14}$ However, these outcome measures may lack the sensitivity to determine graft maturity. ${ }^{11,15}$ Histologic analysis of biopsy graft specimens during second-look arthroscopy is considered the gold standard to determine graft maturity. However, this method is invasive and, therefore, not ideal for clinical follow-up. ${ }^{4}$ Magnetic resonance imaging (MRI) is noninvasive and potentially suitable to assess graft maturity longitudinally in vivo and guide the time of return to sport. ${ }^{15,16}$ Previous animal studies already demonstrated that larger graft volume and lower graftsignal intensity are correlated with higher strength and superior biomechanical properties of the reconstructed ACL. ${ }^{17-19}$ Likewise, human studies have investigated MRI signal-to-noise quotient (SNQ) and its relation to graft maturity. ${ }^{13,20-26}$

Previous systematic reviews of remodeling focused mainly on human and animal biopsy studies ${ }^{3-5}$ and on graft-maturity assessment using MRI. ${ }^{27}$ The purpose of this investigation was to evaluate systematically the current literature concerning biopsy, MRI SNQ and clinical outcomes in graft maturity assessment after autograft ACLR, and their possible relationships. Our hypothesis is that graft SNQ follows a clear pattern over time and resembles histologic remodeling stages and that a correlation with clinical parameters can be found.

\section{Methods}

\section{Protocol and Registration}

The systematic review was reported and conducted according to the PRISMA (Preferred Reporting Items for Systematic reviews and Meta-Analyses) guidelines. ${ }^{28}$ Study protocol was registered with International Prospective Register of Systematic Reviews (PROSPERO), ID CRD42018094585.

\section{Eligibility criteria}

Studies reporting methods of intra-articular ACL graft maturity assessment, at any time after primary ligament reconstruction surgery using autografts, were considered for inclusion. Eligible methods were histologic studies of biopsy specimens and conventional MRI studies reporting serial signal intensity values and/or correlation of MRI signal intensity with clinical parameters such as knee stability scores and PROMs. Signal intensity had to be normalized to surrounding tissue and/or background noise such as the SNQ or signal-to-noise ratio (SNR). The following exclusion criteria were used: case reports, advanced MRI techniques (e.g., diffusion tensor imaging, angiography), focus on tunnel healing, allografts-only studies, patients younger than 18 years of age, cadaver and animal studies, as well as secondary reconstructions. Language was limited to English, Dutch, German, French, and Portuguese.

\section{Information Sources and Search}

An experienced librarian performed the systematic search, until May 15, 2019, in Embase, MEDLINE ovid, Cochrane Central, Web-of-Science, Scopus, Cinahl EBSCOhost, Sportdiscus EBSCOhost, and Google Scholar. The search strategy employed included the following key terms: "knee ligament reconstruction," "anterior cruciate ligament reconstruction," "autograft," "bone patellar tendon bone graft," "hamstring tendon," "healing," and "remodeling."

\section{Study Selection}

Titles and abstracts of the search results were assessed for eligibility by 3 reviewers (B.v.G., D.M.J., R.P.A.J.). The same reviewers also assessed full texts of this selection for final inclusion. Any disagreement among the reviewers was resolved by discussion, and another reviewer could be consulted in case of nonconsent. All references of the included full texts were manually searched for potentially missed eligible articles.

\section{Data Extraction}

Data were extracted by 1 and verified by another author (B.v.G., D.M.J.). General study and patients' characteristics were extracted for each included study. Furthermore, for the biopsy studies, extracted data consisted of reported healing stages and examined tissue aspects (e.g., cellular aspects, vascularity, extracellular matrix). Data of interest from imaging studies consisted of MRI technique (e.g., magnet strength [Tesla], coil type, sequence, acquisition, slice/voxel size, field of view, and SNQ details). Extracted SNQ value was always selected from the autograft midportion and nonintervention group (if applicable). In addition, outcome and corresponding statistical measures (e.g., $\mathrm{R}$-squared and correlation coefficients) describing the relationship between image and clinical/functional parameters were extracted.

\section{Quality Assessment}

Two reviewers (B.v.G., M.C.v.d.S.) independently assessed the individual studies for risk of bias. The assessment was based on the Cochrane Collaboration tool for assessing risk of bias. ${ }^{29}$ Risk of bias and assessed types of bias are presented in Table 1. Items could be scored as low risk $(+)$, high risk $(-)$, unknown/not reported (?), or not applicable (NA). The assessments by the 2 independent reviewers were compared and discussed for final consensus. Another reviewer could be consulted in case of nonconsent. 
Table 1. Risk of Bas in Included Studies

\begin{tabular}{|c|c|c|c|c|c|c|}
\hline & $\begin{array}{l}\text { Selection Bias } \\
1 \text { (Patient selection) }\end{array}$ & $\begin{array}{c}\text { Selection Bias } 2 \\
\text { (Patient characteristics) }\end{array}$ & $\begin{array}{l}\text { Performance } \\
\text { Bias }\end{array}$ & $\begin{array}{l}\text { Detection } \\
\text { Bias }\end{array}$ & $\begin{array}{l}\text { Attritian } \\
\text { Bias }\end{array}$ & $\begin{array}{c}\text { Reporting } \\
\text { Bias }\end{array}$ \\
\hline \multicolumn{7}{|l|}{ Biopsy studies } \\
\hline Abe (1993) & $?$ & + & + & $?$ & NA & + \\
\hline Dong (2005) & + & + & + & + & NA & $?$ \\
\hline Falconiero (1998) & $?$ & + & + & + & - & ? \\
\hline Janssen (2011) & + & + & + & $?$ & NA & + \\
\hline Sanchez (2010) & + & + & + & + & + & $?$ \\
\hline Zaffagnini (2007) & $?$ & + & + & $?$ & NA & + \\
\hline Zaffagnini (2010) & ? & + & + & ? & NA & + \\
\hline \multicolumn{7}{|c|}{ Imaging studies (serial SNQ) } \\
\hline Chen (2018) & $?$ & + & + & + & + & + \\
\hline Gohil (2007) & + & + & + & + & + & + \\
\hline Stockle (1998) & $?$ & + & + & + & NA & + \\
\hline Tashiro (2017) & $?$ & + & + & $?$ & + & + \\
\hline $\operatorname{Vogl}(2001)$ & - & + & + & $?$ & NA & + \\
\hline \multicolumn{7}{|c|}{ Imaging studies (clinical) } \\
\hline Biercevicz (2015) & ? & + & + & + & $?$ & + \\
\hline Hofbauer (2019) & + & $?$ & + & $?$ & NA & + \\
\hline Li (2014) & $?$ & + & + & $?$ & + & $?$ \\
\hline Li (2017) & $?$ & + & + & + & + & + \\
\hline
\end{tabular}

NA, not applicable; SNQ, signal to noise quotient; +, low risk of bias; -, high risk of bias; ?, unknown/not reported.

\section{Results}

Fig 1 shows the flowchart and details of the systematic search. Table 2 shows the characteristics of 10 included human biopsy studies with a total of 316 grafts. $^{6-9,30-35}$ The majority of included cases were clinically stable after reconstruction. However, none of the included studies compared histologic findings to clinical or functional outcomes. Of the imaging studies, 10 reported serial MRI measurements using SNQ. ${ }^{13,20-24,26,36-38}$ Table 3 shows the study characteristics and MRI parameters of included imaging studies reporting serial SNQ; there was a total of 479 patients. A total of 4 imaging studies reporting on the relationship with clinical parameters could be selected; there was a total of 227 patients (Table 4). 13,15,39,40

Most included studies contained risk of bias (see Table 1 for the quality assessment of included studies), especially risk of selection bias due to the number of retrospective studies and case series. No study showed risk of performance bias. Furthermore, no biopsy study reached a level of evidence higher than 3 (Table 2). For the imaging/clinical studies, the highest level of evidence was 2 (Tables 3 and 4).

The Results section covers the following subjects: (1) histologic appearance of human hamstring tendon (HT) and bone patellar tendon bone (BPTB) autografts; (2) postoperative changes in MRI graft SNQ over time; and
(3) relation of imaging parameters with clinical and functional outcomes.

\section{Histologic appearance of human autografts: summary of relevant findings of included studies}

Fig 2 shows the ligamentization stages as published by Pauzenberger et al., ${ }^{5}$ which was adapted from Claes et al. ${ }^{4}$ The studies show that the postoperative healing process of hamstring and BPTB autografts evolve in a typical progression through 3 distinguishable remodeling phases: an early healing phase, a phase of remodeling and a ligamentization phase. ${ }^{6-9,30}$ Despite variations in the reported duration of remodeling phases, included studies suggest a delayed progression in hamstring grafts compared to BPTB grafts. ${ }^{5}$ In the absence of new studies reporting results according to this timescale, this systematic review summarizes overall relevant histologic findings over time after ACLR, as grouped by cellular aspects, vascularity and extracellular matrix as reported in the included studies. ${ }^{6-9,30-35}$

\section{Cellular aspects}

Cells were predominantly ovoid for the first 12 months after ACLR. ${ }^{8,30}$ Morphology changed to more spindle-shaped and linear cells between 13 and 24 months after reconstruction. ${ }^{8,30}$ After 3 years, cell shape was predominantly narrow and long. ${ }^{7,8}$ 


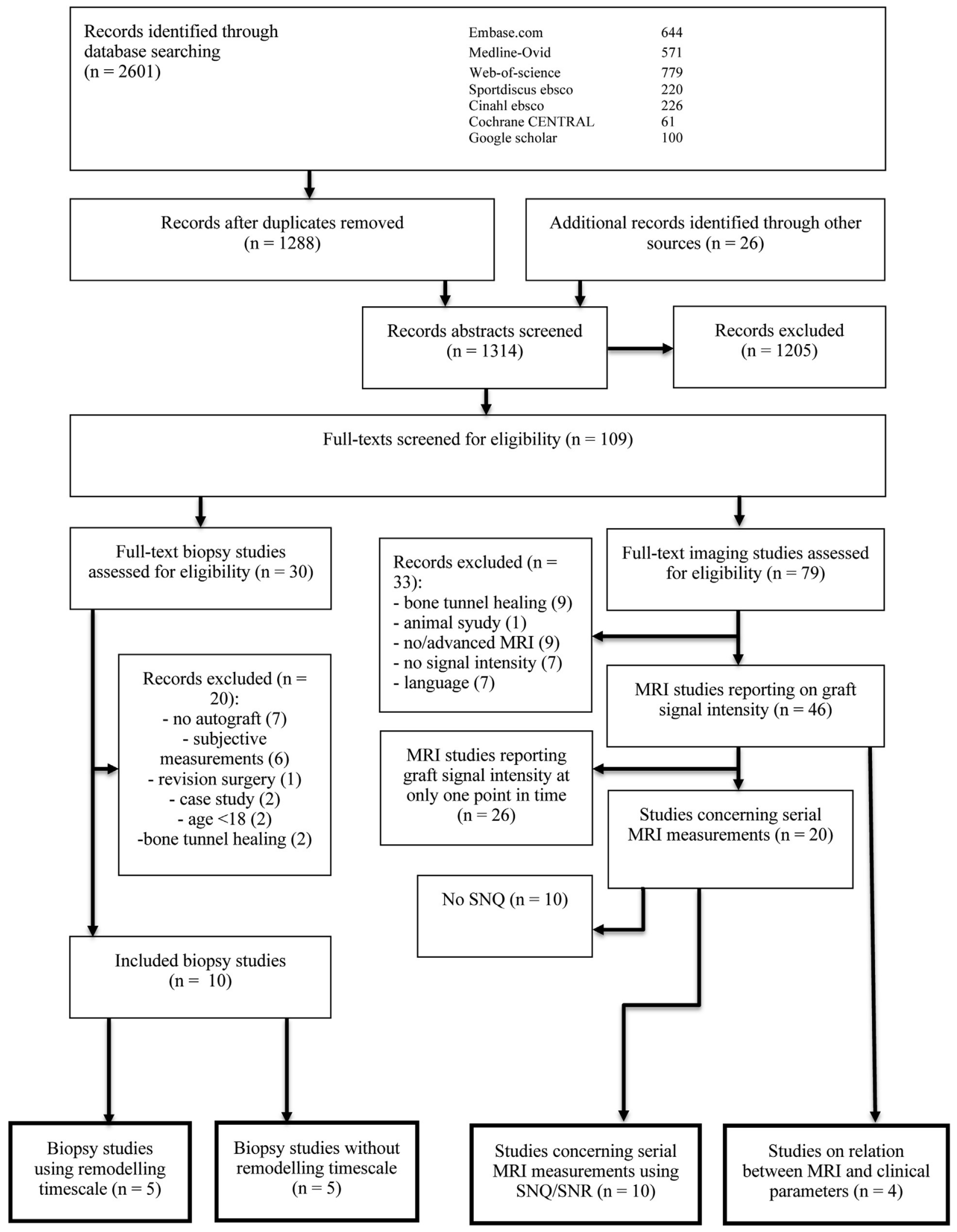

Fig 1. Study flow chart.

Total cell numbers were significantly increased compared to the normal ACL during the first year after ACLR for both HT and BPTB grafts. ${ }^{6-9}$ Abe et al. ${ }^{9}$ showed the presence of so-called metabolically active fibroblasts in their ultrastructure evaluation of BPTB graft biopsies at 6 months and 1 year postoperatively, 
Table 2. Characteristics of Biopsy Studies

\begin{tabular}{|c|c|c|c|c|c|c|c|c|c|c|}
\hline & $\begin{array}{l}\text { Abe } \\
(1993)\end{array}$ & $\begin{array}{l}\text { Cho } \\
(2004)\end{array}$ & $\begin{array}{l}\text { Dong } \\
(2015)\end{array}$ & $\begin{array}{c}\text { Falconiero } \\
(1998)\end{array}$ & $\begin{array}{c}\text { Janssen } \\
(2011)\end{array}$ & $\begin{array}{l}\text { Marumo } \\
(2005)\end{array}$ & $\begin{array}{c}\text { Rougraff } \\
(1993)\end{array}$ & $\begin{array}{l}\text { Sanchez } \\
(2010)\end{array}$ & $\begin{array}{l}\text { Zaffagnini } \\
(2007)\end{array}$ & $\begin{array}{l}\text { Zaffagnini } \\
(2010)\end{array}$ \\
\hline Study design & Case series & Case-control & Case-control & Case-control & Case-control & Case-control & Case series & Case-control & Case-control & $\begin{array}{l}\text { Cross-sectional } \\
\text { study }\end{array}$ \\
\hline $\begin{array}{l}\text { Level of } \\
\text { evidence }\end{array}$ & 4 & 3 & 3 & 3 & 3 & 3 & 4 & 3 & 3 & 3 \\
\hline $\mathrm{N}$ & 21 & 25 & 52 & 48 & 67 & 50 & 23 & 37 & 10 & 8 \\
\hline Female & 15 & 5 vs. 7 & 16 & 15 & 22 & 19 & 3 & 11 & 0 & 0 \\
\hline $\begin{array}{l}\text { Mean age } \\
\quad \text { (range) }\end{array}$ & $14-36$ & $\begin{array}{l}26(18-29) \text { vs. } 25 \\
(15-39)\end{array}$ & $29.0 / 29.6$ & $26.1(16-35)$ & $\begin{array}{l}\text { Group } 1=31.1 ; 2= \\
\quad 27.7 ; 3=29.0\end{array}$ & $\begin{array}{l}28.5(15.1- \\
49.0) \text { vs } 24.5 \\
(13.3-44.0)\end{array}$ & Not mentioned & $28(18-48)$ & $25(18-32)$ & $26(19-31)$ \\
\hline $\begin{array}{l}\text { Follow-up } \\
\text { period }\end{array}$ & 6 wks- 15 mo & $\begin{array}{c}16(7-27) \text { vs. } 20 \\
(9-39) \mathrm{mo}\end{array}$ & $13-62 \mathrm{mo}$ & $3 \mathrm{mo}-10 \mathrm{yrs}$ & 6-117 mo & $11-13 \mathrm{mo}$ & 3 wks- 6.5 yrs & $6-24 \mathrm{mo}$ & $\begin{array}{l}6,12,24,48 \\
120 \mathrm{mo}\end{array}$ & $\begin{array}{l}12,24,48,120 \\
\text { mo }\end{array}$ \\
\hline $\begin{array}{l}\text { Independent } \\
\text { examiner }\end{array}$ & $\begin{array}{l}\text { Not } \\
\text { mentioned }\end{array}$ & Not mentioned & 2 & 2 & Not mentioned & Not mentioned & Not mentioned & 3 (blinded) & Not mentioned & Not mentioned \\
\hline Graft type & ВРТВ & $\begin{array}{l}10 \text { DB 2-strand } \\
\text { (ST) vs } 15 \mathrm{SB} \\
\text { triple/ } \\
\text { quadruple } \\
\text { (ST+/-GT) }\end{array}$ & $\begin{array}{l}\text { DB 8-strand } \\
\text { ST + GT }\end{array}$ & 35 ВРТВ, 8 HS & Quadruple HS & $\begin{array}{l}30 \text { BPTB, } 20 \\
\text { ST }+ \text { GT }\end{array}$ & ВРТВ & HS & ВРТВ & $\begin{array}{c}\text { Quadruple } \\
\text { ST }+ \text { GT }\end{array}$ \\
\hline Clinical outcome & Stable & Not mentioned & $\begin{array}{l}\text { Stable (clinical } \\
\text { failure } \\
\text { excluded) }\end{array}$ & $\begin{array}{l}2 \text { grade III/IV, } 5 \\
\text { grade } 2, \text { and } \\
41 \text { grade I } \\
\text { Lachman }\end{array}$ & Stable & Stable & Stable & Stable & $\begin{array}{l}\text { KT } 2000<3 \\
\text { mm in } 7 \\
\text { cases, } 3-5 \\
\text { mm in } 3 \\
\text { cases }\end{array}$ & $\begin{array}{l}\text { KT } 2000 \text { was }<3 \\
\text { mm in } 6 \text { cases, } \\
3-5 \mathrm{~mm} \text { in } 2 \\
\text { cases }\end{array}$ \\
\hline $\begin{array}{l}\text { Reason for } \\
\text { reoperation }\end{array}$ & HR & HR & HR & New symptoms & HR & HR & $\begin{array}{l}\text { New } \\
\text { pathology, } \\
\text { HR, } \\
\text { voluntary }\end{array}$ & $\begin{array}{l}\text { New } \\
\text { pathology, } \\
\text { HR }\end{array}$ & $\begin{array}{l}\text { Menisectomy } \\
\text { or HR }\end{array}$ & $\begin{array}{l}\text { Menisectomy or } \\
\text { HR }\end{array}$ \\
\hline Biopsy site & $\begin{array}{l}\text { Central } \\
\text { superficial }\end{array}$ & $\begin{array}{l}\text { Central } \\
\text { superficial }\end{array}$ & $\begin{array}{l}\text { Central } \\
\text { (midstrand) }\end{array}$ & $\begin{array}{l}\text { Central } \\
\text { superficial }+ \\
\text { deep }\end{array}$ & Central superficial & $\begin{array}{l}\text { Most central } \\
\text { part }\end{array}$ & $\begin{array}{l}\text { Central } \\
\text { superficial }\end{array}$ & $\begin{array}{l}\text { Directly under } \\
\text { femoral } \\
\text { condyle }\end{array}$ & $\begin{array}{l}\text { Most central } \\
\text { part }\end{array}$ & Most central part \\
\hline $\begin{array}{l}\text { Analysis } \\
\text { technique }\end{array}$ & $\mathrm{LM}+\mathrm{EM}$ & EM & $\mathrm{LM}+\mathrm{EM}$ & LM & $\begin{array}{l}\text { Immunostaining + } \\
\text { LM }\end{array}$ & LM, HPLC & LM & LM & EM & EM \\
\hline Tissue aspects & $\begin{array}{l}\mathrm{V}+\mathrm{C}+\mathrm{F}+ \\
\quad \mathrm{Col}\end{array}$ & Col & $\mathrm{C}+\mathrm{V}+\mathrm{Col}$ & $\mathrm{C}+\mathrm{V}+\mathrm{Col}$ & $\mathrm{V}+\mathrm{C}+\mathrm{F}+\mathrm{Col}$ & Col & $\mathrm{C}+\mathrm{V}+\mathrm{Col}$ & $\mathrm{C}+\mathrm{V}$ & Col & Col \\
\hline
\end{tabular}

BPTB, bone-patellar tendon-bone; C, cellularity; Col, collagen; DB, double bundle; EM, electron microscopy; F, fibroblasts; GT, gracilis tendon; HR, hardware removal; HPLC, highperformance liquid chromatography; HS, hamstring; LM, light microscopy; PRGF, platelet rich growth factor; SB, single bundle; ST, semitendinosus tendon; V, vascularity. 
Table 3. Study Characteristics of Imaging Studies With Serial SNQ Measurements

\begin{tabular}{|c|c|c|c|c|c|c|c|c|c|c|}
\hline & $\begin{array}{l}\text { Chen } \\
(2018)\end{array}$ & $\begin{array}{c}\text { Gohil } \\
(2007) \\
\end{array}$ & $\begin{array}{c}\text { Fukuda } \\
(2019) \\
\end{array}$ & $\begin{array}{c}\text { Lee } \\
(2016) \\
\end{array}$ & $\begin{array}{c}\text { Liu } \\
(2019) \\
\end{array}$ & $\begin{array}{c}\text { Muramatsu } \\
(2008)\end{array}$ & $\begin{array}{l}\text { Stockle } \\
(1998)\end{array}$ & $\begin{array}{l}\text { Tashiro } \\
(2017) \\
\end{array}$ & $\begin{array}{c}\text { Vogl } \\
(2001)\end{array}$ & $\begin{array}{c}\mathrm{Li} \\
(2017)^{*}\end{array}$ \\
\hline Study design & $\begin{array}{c}\text { Prospective } \\
\text { cohort }\end{array}$ & $\begin{array}{l}\text { Randomised } \\
\text { prospective } \\
\text { study }\end{array}$ & Case series & $\begin{array}{l}\text { Retrospective } \\
\text { cohort }\end{array}$ & $\begin{array}{l}\text { Randomized } \\
\text { controlled } \\
\text { trial }\end{array}$ & $\begin{array}{l}\text { Retrospective } \\
\text { comparitive } \\
\text { study }\end{array}$ & Case series & $\begin{array}{c}\text { Prospective } \\
\text { cohort }\end{array}$ & Case series & $\begin{array}{c}\text { Prospective } \\
\text { cohort }\end{array}$ \\
\hline $\begin{array}{l}\text { Level of } \\
\text { evidence }\end{array}$ & 3 & 2 & 4 & 3 & 2 & 3 & 4 & 3 & 4 & 3 \\
\hline $\mathrm{N}$ & 48 & 49 & 45 & 98 & 45 & 44 & 20 & 24 & 68 & 38 \\
\hline Female & Not mentioned & 22 & 27 & 12 & 12 & 8 & 8 & 7 & 33 & 0 \\
\hline $\begin{array}{c}\text { Mean age (SD/ } \\
\text { range) }\end{array}$ & $\begin{array}{l}30( \pm 6) / 29( \pm \\
6)\end{array}$ & $\begin{array}{l}35.5(21-50) / \\
30.5(15-59)\end{array}$ & $27( \pm 8.5)$ & $30.1 / 30.4$ & $31.5 / 29.4$ & $\begin{array}{l}26.1( \pm 1.6) / \\
26.5( \pm 1.2)\end{array}$ & $30(17-59)$ & $20( \pm 4)$ & $36(17-59)$ & $\begin{array}{l}30.8( \pm 5.9) / \\
29.5( \pm 5.0)\end{array}$ \\
\hline Graft type $^{\dagger}$ & $\begin{array}{c}\mathrm{ST}+\mathrm{GT} \\
\text { autograft, } \\
\text { allograft }\end{array}$ & $\begin{array}{c}\text { Double-loop } \\
\text { ST + GT } \\
\text { autograft }\end{array}$ & $\begin{array}{l}\text { Double bundle } \\
\text { ST/GT } \\
\text { autograft }\end{array}$ & $\begin{array}{l}\text { 4-strand ST }+ \\
\text { GT autograft }\end{array}$ & $\begin{array}{l}\text { 4-strand ST }+ \\
\text { GT autograft }\end{array}$ & $\begin{array}{l}\text { ВРТВ } \\
\quad \text { autograft }+ \\
\text { allograft }\end{array}$ & BPTB autograft & $\begin{array}{l}\text { Quadriceps } \\
\text { tendon } \\
\text { autograft }\end{array}$ & BPTB autograft & $\begin{array}{l}\text { 4-strand ST/GT } \\
\text { autograft, } \\
\text { tibialis } \\
\text { anterior } \\
\text { allograft }\end{array}$ \\
\hline MRI follow-up & $3,6,12 \mathrm{mo}$ & $2,6,12 \mathrm{mo}$ & $\begin{array}{l}3 \text { wks, 3, 6, 9, } \\
12,18,24 \\
36,48,50 \\
\text { mo }\end{array}$ & $\begin{array}{l}4 \text { periodes, up to } \\
18 \text { mo }\end{array}$ & $3,6,12,24 \mathrm{mo}$ & $1,4,6,12 \mathrm{mo}$ & $\begin{array}{c}2,12,24,52 \\
102 \text { wks }\end{array}$ & $6,24 \mathrm{mo}$ & $\begin{array}{c}2,12,52,76 \\
104 \mathrm{wks}\end{array}$ & $3,6,12 \mathrm{mo}$ \\
\hline $\begin{array}{l}\text { Magnet strength } \\
\text { (Tesla) }\end{array}$ & 3.0 & 1.5 & 0.3 & 1.5 & 3.0 & 1.0 & 1.5 & 3.0 & 1.5 & 3.0 \\
\hline Coil type & Not mentioned & Knee coil & Not mentioned & Extremity coil & Not mentioned & Not mentioned & Knee coil & Not mentioned & Extremity coil & Not mentioned \\
\hline Sequence & PD FS & PD FSE & PD SE & $\mathrm{PD}$ & FD FS & T1 Gd-DTPA & $\begin{array}{c}\text { T1/T2 SE + T1 } \\
\text { Gd-DTPA }\end{array}$ & $\begin{array}{l}\text { DESS, short TE } \\
\text { T2 }\end{array}$ & $\begin{array}{l}\text { PD SE Tl/T2, Tl } \\
\text { SE/FS Gd- } \\
\text { DTPA }\end{array}$ & PD FS \\
\hline $\begin{array}{l}\text { Acquisition } \\
\text { parameters } \\
\text { (TR/TE) } \\
\text { (ms) }\end{array}$ & Not mentioned & $3000 / 30$ & $1800 / 20$ & $\begin{array}{l}3000-4000 / 17- \\
18\end{array}$ & $3000 / 28$ & $500 / 17$ & NA & $\begin{array}{l}16.3 / 4.7-2700 / \\
\quad 10.6\end{array}$ & $\begin{array}{l}800 / 5 \\
500 / 15\end{array}$ & $3000 / 28$ \\
\hline $\begin{array}{l}\text { MRI slice } \\
\text { thickness } \\
(\mathrm{mm})\end{array}$ & Not mentioned & 2 & 4 & Not mentioned & 3 & Not mentioned & Not mentioned & 3 & 3 & 3 \\
\hline $\begin{array}{l}\text { MRI voxel size } \\
(\mathrm{mm})\end{array}$ & Not mentioned & Not mentioned & Not mentioned & Not mentioned & Not mentioned & Not mentioned & Not mentioned & $\begin{array}{l}0.45 \times 0.45 \times \\
0.70\end{array}$ & Not mentioned & Not mentioned \\
\hline $\begin{array}{l}\text { Field of view } \\
\qquad(\mathrm{mm})\end{array}$ & Not mentioned & Not mentioned & 170 & Not mentioned & 150 & Not mentioned & Not mentioned & Not mentioned & $200 / 400$ & 150 \\
\hline SNQ calculation & $\begin{array}{l}\text { (SI graft-SI } \\
\text { PCL)/SI } \\
\text { background }\end{array}$ & $\begin{array}{l}\text { SI graft/SI } \\
\text { background }\end{array}$ & $\begin{array}{l}\text { (SI graft-SI } \\
\text { PCL)/SI } \\
\text { background }\end{array}$ & $\begin{array}{l}\text { (SI graft-SI } \\
\text { quadriceps } \\
\text { tendon)/SI } \\
\text { background }\end{array}$ & $\begin{array}{l}\text { (SI graft-SI } \\
\text { quadriceps } \\
\text { tendon)/SI } \\
\text { background }\end{array}$ & $\begin{array}{l}\text { (SI graft-SI } \\
\text { quadriceps } \\
\text { tendon)/SI } \\
\text { background }\end{array}$ & $\begin{array}{l}\text { (SI graft-SI } \\
\text { patellar } \\
\text { tendon)/SI } \\
\text { background }\end{array}$ & $\begin{array}{l}\text { (SI graft-SI } \\
\text { PCL)/SI } \\
\text { background }\end{array}$ & $\begin{array}{l}\text { (SI graft-SI } \\
\text { quadriceps } \\
\text { tendon)/SI } \\
\text { background }\end{array}$ & $\begin{array}{l}\text { (SI graft-SI } \\
\text { quadriceps } \\
\text { tendon)/SI } \\
\text { background }\end{array}$ \\
\hline SNQ zones & 4 & 4 & $\begin{array}{l}1 \text { site each } \\
\text { bundle }\end{array}$ & 3 & 3 & 1 & 3 & 3 & 3 & 3 \\
\hline
\end{tabular}

hamstring; MRI, magnetic resonance imaging; PCL, posterior cruciate ligament; PD, proton density; SI, signal intensity; SNQ, signal to noise quotient; ST, semitendinosus tendon; TR, time-torepetition.

*Same study as mentioned in Table 4.

${ }^{\dagger}$ Only autograft data was used in the analyses. 
Table 4. Study Characteristics of Imaging/Clinical Studies

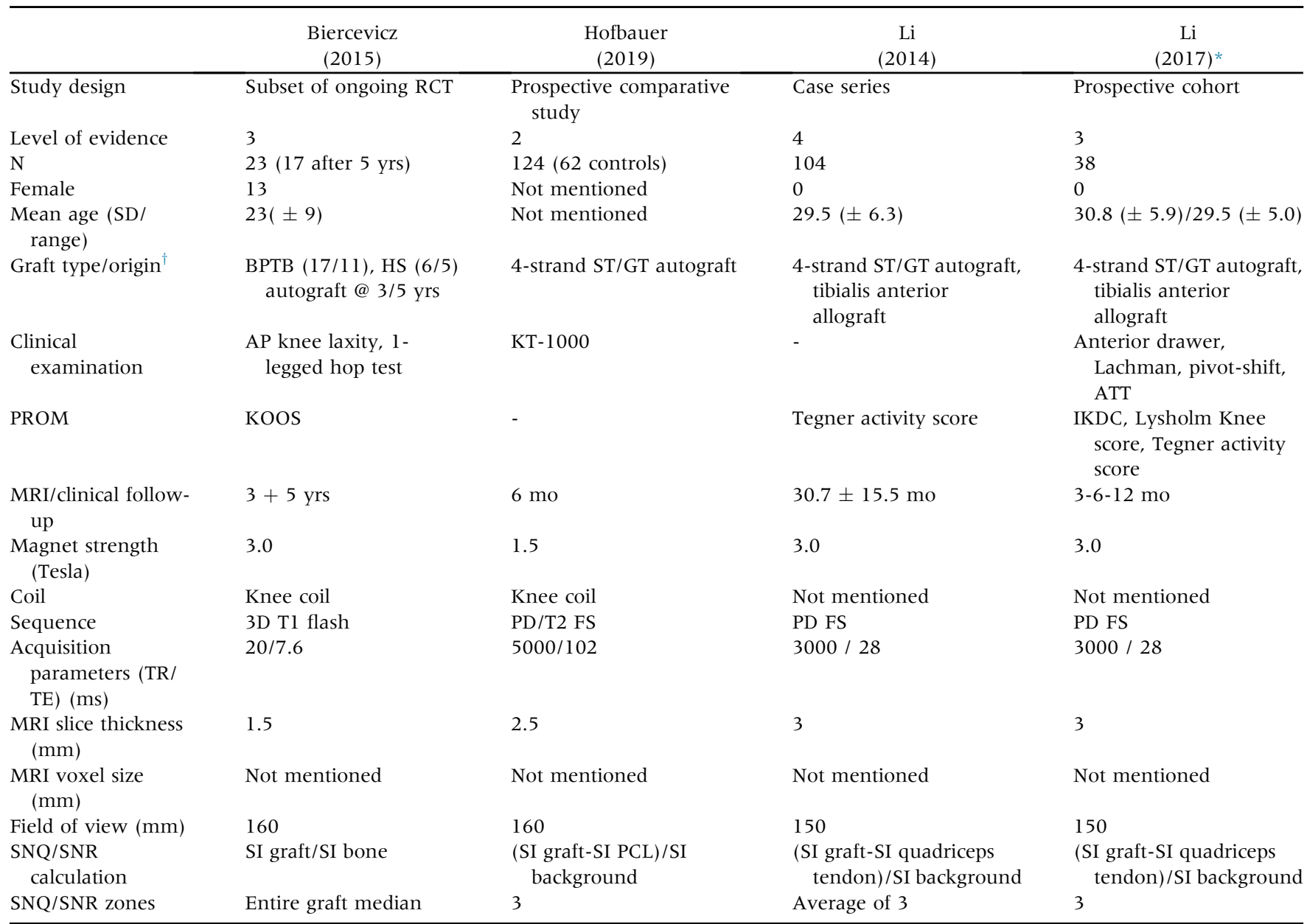

ATT, anterior tibial translation; BPTB, bone-patellar tendon-bone; FS, fat saturated; GT, gracilis tendon; IKDC, international knee documentation committee score; KOOS, knee injury and osteoarthritis outcome score; MRI, magnetic resonance imaging; NA, not available; PCL, posterior cruciate ligament; PD, proton density; PROM, patient reported outcome measure; RCT, randomized controlled trial; SI, signal intensity; SNQ, signal to noise quotient; SNR, signal to noise ration; ST, semitendinosus tendon; TR, time-to-repetition.

${ }^{*}$ Same study as mentioned in Table 3 .

${ }^{\dagger}$ Only autograft data was used in the analyses.

indicating ongoing remodeling. Janssen et al. ${ }^{8}$ found a strong increase in myofibroblast density from 13 up to 24 months after ACLR in HT grafts. This indicated an active remodeling process for HS grafts even 1 to 2 years after surgery. ${ }^{8}$

Dong et al., ${ }^{33}$ in their biopsy study of double-bundle ACL reconstruction using hamstring autografts, found no statistical difference in cellularity, metaplasia or cellular metabolic status between the midterm group assessed 13 to 30 months after ACLR and the long-term group assessed 31 to 62 months after ACLR. No data were presented for the first year after ACLR.

\section{Vascularity}

Hypervascularity characterized the early healing stage (between 6 and 8 weeks), during which the graft is covered with thick synovial tissue containing abundant capillary blood vessels. ${ }^{9}$ Rougraff et al. ${ }^{7}$ found a trend of increased neovascularity in the BPTB graft biopsies during the first 2 years after ACLR, toward a gradual decrease in the later biopsies up to 78 months after reconstruction. Janssen et al. ${ }^{8}$ also reported an increase in vessel density in biopsy groups, but it never reached the level of normal ACL at any time during the 117 months of follow-up. In contrast, Falconiero et al. ${ }^{6}$ reported that biopsied portions of HT and BPTB grafts 12 months after ACLR closely resembled the normal ACL, indicating vascular maturity.

\section{Extracellular matrix}

From the early stage of the postoperative period at 68 weeks $^{9}$ up to 12 months after ACLR, ${ }^{7,8}$ biopsy studies showed an irregular collagen orientation. After this first period, it changed into a more regular orientation, adapting to but not fully restoring the appearance of a normal ACL. ${ }^{6,8}$ 
Ligamentization of human tendon grafts

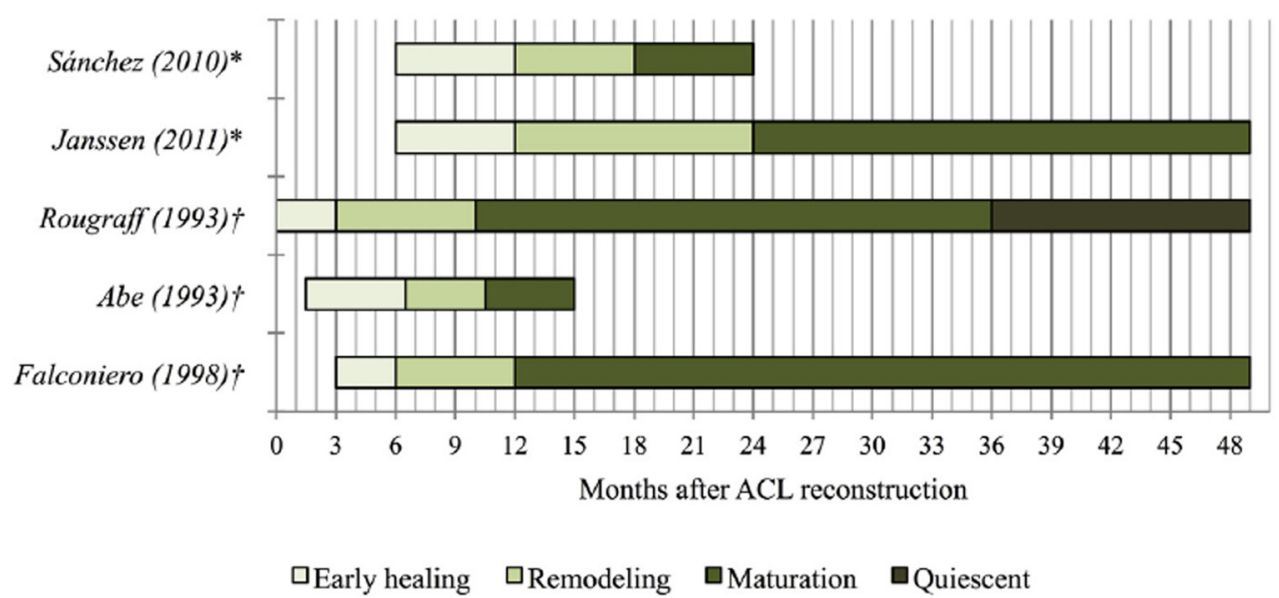

Fig 2. Ligamentization stages in human hamstring $(*)$ and patellar $(\dagger)$ tendon grafts, as published by Pauzenberger et al. ${ }^{5}$ ACL, anterior cruciate ligament.

$*=$ hamstring tendon grafts

$\dagger=$ patellar tendon grafts

Tendinous tissue had a considerably different collagen-reducible crosslinks elution profile compared to ligamentous tissue. The cruciate ligaments showed the highest ratio between dihydroxylysinonorleucine (DHLNL) and hydroxylysinonorleucine (HLNL) when compared to patellar tendons or semitendinosus and gracilis tendons. ${ }^{34}$ Marumo et al. ${ }^{34}$ found an increase in this DHLNL/HLNL ratio over time and patterns of reducible crosslinks in BPTB and HT autografts resembling the normal ACL approximately 1 year after surgery.

Cho et al. ${ }^{31}$ compared electron microscopy collagen findings in double-bundle and single-bundle hamstring tendon autografts. The diameter of collagen fibrils in the reconstructed ACL with hamstring tendons was significantly smaller than that in the original hamstring tendons. Moreover, collagen fibril diameter was significantly larger in the double-bundle group, possibly leading to increased tensile strength., ${ }^{9,31}$

Large-diameter collagen fibrils commonly decreased over time and eventually disappeared, leading to a change in collagen distribution from a bimodal to a unimodal pattern in ACLR using allografts and autografts. ${ }^{9,32,35}$ These ultrastructural changes occurred mainly during the first 2 years after ACLR for both hamstring and BPTB grafts. No evident further changes took place from this moment until 10 years after surgery. ${ }^{32,35}$ In contrast, Dong et al. ${ }^{33}$ found a higher percentage of large-diameter collagen fibrils and a bimodal distribution, not changing significantly between the midterm group, 13-30 months after ACLR, and the long-term group, 31-62 months after ACLR.

\section{Postoperative changes in MRI graft signal to noise quotient over time}

SNQ was measured using a region-of-interest tool on a single image (mostly oblique coupes) in all cases. As seen in Tables 3 and 4, different SNQ/SNR calculation techniques were used across the included studies. Despite variation in absolute values, the included studies showed an initial increase of the SNQ/SNR in the postoperative period, peaking at approximately 6 months, followed by a gradual decrease over time toward direct postoperative values. ${ }^{13,20-24,26,36-38}$ None of the included studies included a correlation of graft SNQ and histologic findings of remodeling.

Two studies reported on the relation between SNQ and the graft bending angle. ${ }^{20,24}$ Tashiro et al. ${ }^{24}$ determined the in vivo dynamic graft bending angles and SNQ at 6 and 24 months after anatomic ACLR using quadriceps tendon autografts in 24 patients. A steep graft bending angle was significantly correlated with high signal intensities of the proximal graft, indicating a possible negative effect on proximal graft healing. At 24 months, mean SNQ had decreased, and no difference was found between graft regions. ${ }^{24}$

Of the included studies, 2 reported on differences in SNQ between for ACLR in combination with ACL remnant preservation. ${ }^{21,22}$ Gohil et al. ${ }^{21}$ compared minimal debridement of the intercondylar notch and the residual ACL stump with a standard technique using HS autografts for ACLR in a randomized controlled trial. The minimal debridement group had significantly higher SNQ at 2 months, whereas the standard group had higher signals at 6 months. At 1 year, there was no significant difference between the groups. ${ }^{21}$ Lee et al. ${ }^{22}$ 
found higher SNQ in the first 2 periods until 4 months after ACLR in their patients in whom the ACL remnant was preserved. In period 3 (6-9 months) there was no statistical difference between the remnant-preserving or -sacrificing group. In period 4 (12-19 months) the SNQ of the remnant sacrificing group was significantly higher. $^{22}$

\section{Relation of imaging parameters with clinical and functional outcome}

Biercevicz et al. ${ }^{15}$ examined the relationship between a combination of graft volume/signal intensity and clinical parameters by means of multiple linear regression models for both BPTB and HT autografts. No data were presented for the first 3 years after ACLR. At 3 years of follow-up, volume combined with median graft signal intensity did not predict any item of the Knee Injury and Osteoarthritis Outcome Score (KOOS). However, at 5 years of follow-up, the combination of volume and signal intensity did predict KOOS-QoL $\left(\mathrm{R}^{2}=0.0 .49, P=0.012\right)$, KOOS-Sport $\left(\mathrm{R}^{2}=0.37, P=0.048\right)$, KOOS-Pain $\left(\mathrm{R}^{2}=\right.$ $0.46, P=0.017)$, and KOOS-Sym $\left(\mathrm{R}^{2}=0.45, P=0.021\right)$. The combination of volume and signal intensity also predicted hop percentage at 3 years $\left(\mathrm{R}^{2}=0.40, P=\right.$ $0.0 .008)$ and 5 years $\left(\mathrm{R}^{2}=0.62, P=0.003\right)$ of follow-up. A study by Hofbauer et al. ${ }^{39}$ reported only a poor correlation between mean SNQ and KT-1000 findings with both Pearson and Spearman coefficients (not specified) at 6 months after ACLR using HS autografts in 62 patients. Li et al. ${ }^{13}$ used Spearman correlation analysis to investigate possible associations among several factors (International Knee Documentation Committee score, Lysholm score, Tegner score, and anterior tibial translation difference), and the MRI SNQ value of HT autografts and tibialis anterior allograft. For the autograft, the graft SNQ had a significant positive association with the anterior tibial translation difference at 3 months $(P=$ $0.01)$. However, no other associations were found between the SNQ and clinical scores at 3, 6 and 12 months after ACLR. In a former study by the same authors, a positive correlation $(\mathrm{r}=0.392, P=0.01)$ was found between graft SNQ and the Tegner score. ${ }^{40}$

\section{Discussion}

Although active remodeling after ACLR continues beyond 1 year after surgery, MRI signal/noise changes over time do not seem to reflect through biopsy determined remodeling stages, nor clinical and functional outcomes.

The systematic review by Claes et al. ${ }^{4}$ was the first to compare remodeling time frames in human grafts after ACLR. Pauzenberger et al. ${ }^{5}$ adapted this idea and added 1 new human study. ${ }^{8}$ Both reviews reported 3 phases of remodeling, consisting of an early healing phase, a phase of remodeling and a maturation or ligamentization phase. Furthermore, they proposed a slower progression of remodeling in HT compared with BPTB autografts. However, interpretation of these results remains difficult due to a lack of strictly defined criteria for remodeling and to differences in study design and surgical techniques. ${ }^{5}$ Compared to Pauzenberger et al., ${ }^{5}$ additional biopsy studies included in this review ${ }^{31-35}$ were evaluated in a qualitative analysis on various histologic aspects (cellularity, vascularity and extracellular matrix of the human ACL graft). Although the majority of included cases were reported to be clinically stable after reconstruction, no correlation was made with histologic findings. However, based on the histologic appearance of active fibroblasts, increased vascularity and collagen fibril diameter changes, there seems to be a trend toward ongoing remodeling even beyond 1 year after ACLR. ${ }^{5,8,9,34,35}$ This finding could indicate insufficient mechanical properties of the graft in the first year after surgery despite clinically stable knee outcome scores and, thus, the need for an extended rehabilitation program in order to reduce the risk of ACL graft rerupture. To guide individual patients toward their optimal timing in return to sport, monitoring the remodeling process is desirable. However, the invasive nature of the biopsy procedure prevents safe and serial observation. Another potential limitation of human biopsies is the tendency to perform mainly superficial graft examination, which could influence the results because it might not represent the whole graft. ${ }^{4,5,34}$ Therefore, a need exists for less invasive techniques to monitor individual graft maturity after ACLR.

MRI-derived parameters predict ex vivo structural properties of the graft after ACLR in animal studies. ${ }^{17-19}$ However, in vitro biomechanical testing of the graft after reconstruction is not possible in humans. As a noninvasive imaging modality, MRI could potentially aid in the indirect assessment of graft maturity after ACLR. MRI is a widely used clinical tool to monitor the graft qualitatively after ACLR. ${ }^{41}$ Signal intensity, an MRI parameter for the function of tissue type and water content, has been used to evaluate the integrity and maturation of the graft. ${ }^{42}$ Signal changes over time represent the gradual reduction in water content and vascularity, as shown in animal studies. These changes possibly reflect the progression of remodeling. ${ }^{19,43} \mathrm{~A}$ growing number of studies report SNQ using background signal, instead of signal intensity alone, to take into account differing physiologic conditions such as tissue hydration. ${ }^{25,27}$ Furthermore, as a normalized quantification of signal intensity, the SNQ is able to compare values more reliably between patients and successive scans. Therefore, SNQ potentially reflects graft maturity better. A pattern of SNQ changes over time could be compared to biopsy findings and could even aid in the determination of graft maturity and, therewith, the timing of return to sport. The included studies all showed an initial increase in SNQ, peaking at 
approximately 6 months, followed by a gradual decrease over time. However, various other underlying causes for change in MRI signal intensity should be considered. Graft type, graft bending angle and associated impingement, and ACL remnant preservation may affect graft healing and, therefore, graft signal intensity, as noted in the Results section. ${ }^{13,20-24,42,44}$ Furthermore, the signal intensity of the graft can vary depending on scanner hardware and MRI acquisition characteristics. $^{45,46}$ For example, higher MRI field strength (Tesla) generates higher SNQ. ${ }^{47}$ Single-image SNR calculation and artefacts due to the orientation variation in MRI signals are 2 more limitations when using the SNQ/SNR for graft maturity assessment. ${ }^{27,46,48}$ Together with differing surgical techniques and the inconsistent intervals of signal-intensity measurements of the included studies, direct comparison of the SNQ values among studies and the graft types used is complicated and statistically questionable. Therefore, no meta-analysis was performed in this systematic review. Furthermore, the postoperative SNQ pattern over time does not seem to reflect the histologic stages of remodeling reported by the studies included in Pauzenberger et al. ${ }^{5}$ To wit, hamstring autograft studies start their early healing phase at 6 months, whereas in BPTB autograft studies, 6 months would still reflect ongoing remodeling or early healing. Together with the above-mentioned limitations of graft SNQ, this impedes the finding of a concrete correlation between graft SNQ and histologic remodeling stages. No single study used both MRI and biopsy methods, so the future place of MRI SNQ in graft maturity assessment after ACLR is uncertain. More research using larger patient numbers and consistent imaging protocols at fixed follow-up times is needed to compare SNQ changes with histologic remodeling time frames as used in human biopsy studies. Also, feasibility studies of $\mathrm{T} 2$ relaxation time and diffusion tensor imaging offer potential possibilities in graft-maturity assessment. ${ }^{11,49,50}$ The results of our study are in line with a review by Van Dyck et al., ${ }^{27}$ who reported a more in-depth analysis of MRI signal intensity feasibility and confounders. In the present review, we were able to include 3 more MRI-related $\operatorname{articles}^{22,24,36}$ and provide an overview of various methods of graft maturity assessment and their relationship.

Although clinical and functional outcome measures are used to evaluate outcome and timing of return to sports after knee ligament reconstruction, there is a lack of objective criteria regarding these tests and their ability to predict graft maturity after ACLR. The determination of graft maturity based on histologic findings relies on multiple parameters. ${ }^{6-9,22,24,30-35}$ Therefore, clinical and functional outcomes are probably not able to predict graft maturity and structural properties directly on the basis of histologic findings. Because MRI parameters predict animal graft maturity and structural properties, ${ }^{17-19}$ these imaging determinations could potentially serve as an indirect measure of graft maturity after ACLR. According to Li et al., ${ }^{13}$ graft SNQ does not have the ability to predict the clinical or in vivo outcome measures in patients at l-year follow-up. Graft SNQ shows an inordinate change during the first year after reconstruction, so it might be an unstable time to find a correlation between graft stability and knee function. ${ }^{13}$ The higher subscores of the KOOS in patients with larger graft volumes and lower signal intensity at 5-year follow-up were not found at the 3-year follow-up in the study by Biercevizc et al. ${ }^{15}$ This might be explained by the lack of variability in graft volume and signal intensity at this time point. ${ }^{15}$ Low number of patients with evident knee laxity in general may also contribute to the inability of relating MRI SNQ to knee stability scores. In addition, although different technical aspects of ACLR influence MRI SNQ, clinical outcomes and functional scores tend to show no significant difference in these studies. ${ }^{13,20,21}$ Furthermore, it is worth noting that knee stability and PROMs are also influenced by muscular strength and proprioception, which could be more important than the integrity of the ACLR as assessed by MRI. The studies included in this systematic review did not reveal an evident correlation between graft SNQ and knee-stability outcome scores at the short- and long-term follow-ups after ACLR. This also means that graft SNQ, as it stands, is not a reliable outcome to guide return to sport.

\section{Limitations}

The limitations of the present systematic review emerge mainly from the heterogeneity of the investigated data in both biopsy and imaging studies. As a result, only a descriptive and qualitative analysis of the included studies was possible. This also prevented direct correlation among the differing methods of graft maturity assessment using the today's available literature. A great number of the included studies showed risk of bias, mainly because of retrospective study designs and case series. Our research questions comprised multiple subtopics within the vast amount of literature on graft remodeling. This may have influenced completeness of search results. However, by keeping the initial search broad and through the support of various languages and the manual searches of the references used in the selected full-text studies, we believe bias was kept to a minimum. Furthermore, as an issue inherent to systematic reviews, publication bias might have been an issue.

\section{Conclusions}

The remodeling of the graft is an ongoing process, even 1 year after ACLR, based on human biopsy studies. MRI SNQ peaked at approximately 6 months, 
followed by a gradual decrease over time. Heterogeneity of the MRI methods and technical restrictions used in the current literature limit prediction of graft maturity, clinical and functional outcome measures by means of MRI graft SNQ after ACLR.

\section{Acknowledgments}

Special thanks are extended to M. Reijman and W.M. Bramer (information specialist) of Erasmus MC Rotterdam for carrying out the search.

\section{References}

1. Schindler OS. Surgery for anterior cruciate ligament deficiency: A historical perspective. Knee Surg Sports Traumatol Arthrosc 2012;20:5-47.

2. Amiel D, Kleiner JB, Roux RD, Harwood FL, Akeson WH. The phenomenon of ligamentization: Anterior cruciate ligament reconstruction with autogenous patellar tendon. J Orthop Res 1986;4:162-172.

3. Janssen RP, Scheffler SU. Intra-articular remodelling of hamstring tendon grafts after anterior cruciate ligament reconstruction. Knee Surg Sports Traumatol Arthrosc 2014;22:2102-2108.

4. Claes S, Verdonk P, Forsyth R, Bellemans J. The "ligamentization" process in anterior cruciate ligament reconstruction: What happens to the human graft? A systematic review of the literature. Am J Sports Med $2011 ; 39: 2476-2483$.

5. Pauzenberger L, Syré S, Schurz M. "Ligamentization" in hamstring tendon grafts after anterior cruciate ligament reconstruction: A systematic review of the literature and a glimpse into the future. Arthroscopy 2013;29:1712-1721.

6. Falconiero RP, DiStefano VJ, Cook TM. Revascularization and ligamentization of autogenous anterior cruciate ligament grafts in humans. Arthroscopy 1998;14:197-205.

7. Rougraff B, Shelbourne KD, Gerth PK, Warner J. Arthroscopic and histologic analysis of human patellar tendon autografts used for anterior cruciate ligament reconstruction. Am J Sports Med 1993;21:277-284.

8. Janssen RPA, van der Wijk J, Fiedler A, et al. Remodelling of human hamstring autografts after anterior cruciate ligament reconstruction. Knee Surg Sports Traumatol Arthroscopy 2011;19:1299-1306.

9. Abe S, Kurosaka M, Iguchi T, et al. Light and electron microscopic study of remodeling and maturation process in autogenous graft for anterior cruciate ligament reconstruction. Arthroscopy 1993;9:394-405.

10. Harris JD, Abrams GD, Bach BR, et al. Return to sport after ACL reconstruction. Orthopedics 2014;37:e103-e108.

11. Van Dyck P, Froeling M, De Smet E, et al. Diffusion tensor imaging of the anterior cruciate ligament graft. J Magn Reson Imag 2017;46:1423-1432.

12. Ardern CL, Taylor NF, Feller JA, Webster KE. Fifty-five percent return to competitive sport following anterior cruciate ligament reconstruction surgery: An updated systematic review and meta-analysis including aspects of physical functioning and contextual factors. Br J Sports Med 2014;48:1543-1552.
13. Li H, Chen JW, Li HY, et al. MRI-based ACL graft maturity does not predict clinical and functional outcomes during the first year after ACL reconstruction. Knee Surg Sports Traumatol Arthrosc 2017;25:3171-3178.

14. Barber-Westin SD, Noyes FR. Objective criteria for return to athletics after anterior cruciate ligament reconstruction and subsequent reinjury rates: A systematic review. Phys Sportsmed 2011;39:100-110.

15. Biercevicz AM, Akelman MR, Fadale PD, et al. MRI volume and signal intensity of ACL graft predict clinical, functional, and patient-oriented outcome measures after ACL reconstruction. Am J Sports Med 2015;43:693-699.

16. Howell SM, Berns GS, Farley TE. Unimpinged and impinged anterior cruciate ligament grafts: MR signal intensity measurements. Radiology 1991;179:639-643.

17. Biercevicz AM, Miranda DL, Machan JT, et al. In situ, noninvasive, $\mathrm{T} 2$ *-weighted MRI-derived parameters predict ex vivo structural properties of an anterior cruciate ligament reconstruction or bioenhanced primary repair in a porcine model. Am J Sports Med 2013;41:560-566.

18. Fleming BC, Vajapeyam S, Connolly SA, et al. The use of magnetic resonance imaging to predict ACL graft structural properties. J Biomech 2011;444:2843-2846.

19. Weiler A, Peters G, Maurer J, et al. Biomechanical properties and vascularity of an anterior cruciate ligament graft can be predicted by contrast-enhanced magnetic resonance imaging: A two-year study in sheep. Am J Sports Med 2001;29:751-761.

20. Chen L, Wu Y, Lin G, et al. Graft bending angle affects allograft tendon maturity early after anterior cruciate ligament reconstruction. Knee Surg Sports Traumatol Arthrosc 2018;10:3048-3054.

21. Gohil S, Annear PO, Breidahl W. Anterior cruciate ligament reconstruction using autologous double hamstrings: A comparison of standard versus minimal debridement techniques using MRI to assess revascularisation: A randomised prospective study with a one-year follow-up. J Bone Joint Surg 2007;89:1165-1171.

22. Lee BI, Kim BM, Kho DH, et al. Does the tibial remnant of the anterior cruciate ligament promote ligamentization? Knee 2016:23:1133-1142.

23. Muramatsu K, Hachiya Y, Izawa H. Serial evaluation of human anterior cruciate ligament grafts by contrastenhanced magnetic resonance imaging: Comparison of allografts and autografts. Arthroscopy 2008;24:1038-1044.

24. Tashiro Y, Gale T, Sundaram V, et al. The graft bending angle can affect early graft healing after anterior cruciate ligament reconstruction: In vivo analysis with 2 years' follow-up. Am J Sports Med 2017;45:1829-1836.

25. Vogrin M, Rupreht M, Dinevski D, et al. Effects of a platelet gel on early graft revascularization after anterior cruciate ligament reconstruction: A prospective, randomized, double-blind, clinical trial. Eur Surg Res 2010;45: 77-85.

26. Vogl TJ, Schmitt J, Lubrich J, et al. Reconstructed anterior cruciate ligaments using patellar tendon ligament grafts: Diagnostic value of contrast-enhanced MRI in a 2-year follow-up regimen. Eur Radiol 2001;11:1450-1456.

27. Van Dyck P, Zazulia K, Smekens C, et al. Assessment of anterior cruciate ligamentgraft maturity with conventional magnetic resonance imaging: A systematic 
literature review. Orthop J Sports Med 2019;7: 2325967119849012.

28. Moher D, Shamseer L, Clarke M, et al. Preferred reporting items for systematic review and meta-analysis protocols (PRISMA-P) 2015 statement. Systemat Rev 2015;4:1.

29. Higgins JPTG, S. (eds.). Cochrane Handbook for Systematic Reviews of Interventions Version 5.1.0 [updated March 2011]. The Cochrane Collaboration; 2011. Available from http://handbook.cochrane.org.

30. Sánchez M, Anitua E, Azofra J, et al. Ligamentization of tendon grafts treated with an endogenous preparation rich in growth factors: Gross morphology and histology. Arthroscopy 2010;26:470-480.

31. Cho S, Muneta T, Ito S, et al. Electron microscopic evaluation of two-bundle anatomically reconstructed anterior cruciate ligament graft. J Orthop Sci 2004;9: 296-301.

32. Zaffagnini S, De Pasquale V, Marchesini Reggiani L, et al. Neoligamentization process of BTPB used for ACL graft: Histological evaluation from 6 months to 10 years. Kne 2007; 14:87-93.

33. Dong S, Xie G, Zhang Y, et al. Ligamentization of autogenous hamstring grafts after anterior cruciate ligament reconstruction: Midterm versus long-term results. Am J Sports Med 2015;43:1908-1917.

34. Marumo K, Saito M, Yamagishi T, Fujii K. The "ligamentization" process in human anterior cruciate ligament reconstruction with autogenous patellar and hamstring tendons: A biochemical study. Am J Sports Med 2005;33: 1166-1173.

35. Zaffagnini S, de Pasquale V, Reggiani LM, et al. Electron microscopy of the remodelling process in hamstring tendon used as ACL graft. Knee Surg Sports Traumatol Arthrosc 2010;18:1052-1058.

36. Fukuda H, Asai S, Kanisawa I, et al. Inferior graft maturity in the PL bundle after autograft hamstring double-bundle ACL reconstruction. Knee Surg Sports Traumatol Arthrosc 2019;27:491-497.

37. Liu S, Li H, Tao $\mathrm{H}$, et al. A randomized clinical trial to evaluate attached hamstring anterior cruciate ligament graft maturity with magnetic resonance imaging. Am J Sports Med 2018;46:1143-1149.

38. Stockle U, Hoffmann R, Schwedke J, et al. Anterior cruciate ligament reconstruction: The diagnostic value of MRI. Int Orthop 1998;22:288-292.

39. Hofbauer M, Soldati F, Szomolanyi P, et al. Hamstring tendon autografts do not show complete graft maturity 6 months postoperatively after anterior cruciate ligament reconstruction. Knee Surg Sports Traumatol Arthrosc 2019;27:130-136.

40. Li H, Chen S, Tao H, et al. Correlation analysis of potential factors influencing graft maturity after anterior cruciate ligament reconstruction. Orthop J Sports Med 2014;2: 2325967114553552.

41. Miller TT. MR imaging of the knee. Sports Med Arthrosc Rev 2009; 17:56-67.

42. Ahn JH, Jeong HJ, Lee YS, et al. Graft bending angle is correlated with femoral intraosseous graft signal intensity in anterior cruciate ligament reconstruction using the outside-in technique. Knee 2016;23:666-673.

43. Howell SM, Knox KE, Farley TE, Taylor MA. Revascularization of a human anterior cruciate ligament graft during the first two years of implantation. Am J Sports Med 1995;23:42-49.

44. Ahn JH, Lee SH, Choi SH, Lim TK. Magnetic resonance imaging evaluation of anterior cruciate ligament reconstruction using quadrupled hamstring tendon autografts: Comparison of remnant bundle preservation and standard technique. Am J Sports Med 2010;38:1768-1777.

45. Deoni SCL, Williams SCR, Jezzard P, et al. Standardized structural magnetic resonance imaging in multicentre studies using quantitative $\mathrm{T} 1$ and $\mathrm{T} 2$ imaging at $1.5 \mathrm{~T}$. NeuroImage 2008;40:662-671.

46. Dietrich O, Raya JG, Reeder SB, et al. Measurement of signal-to-noise ratios in MR images: influence of multichannel coils, parallel imaging, and reconstruction filters. J Magn Reson Imaging 2007;26:375-385.

47. Li H, Tao H, Cho S, et al. Difference in graft maturity of the reconstructed anterior cruciate ligament 2 years postoperatively: A comparison between autografts and allografts in young men using clinical and 3.0-T magnetic resonance imaging evaluation. Am J Sports Med 2012;40: 1519-1526.

48. Echigo J, Yoshioka H, Takahashi H, et al. Signal intensity changes in anterior cruciate ligament autografts: Relation to magnetic field orientation. Academ Radiol 1999;6: 206-210.

49. Beveridge JE, Machan JT, Walsh EG, et al. Magnetic resonance measurements of tissue quantity and quality using $\mathrm{T} 2$ * relaxometry predict temporal changes in the biomechanical properties of the healing ACL. J Orthop Res 2018;36:1701-1709.

50. Liu J, Nazaran A, Ma Y, et al. Single- and bicomponent analyses of $\mathrm{T} 2$ relaxation in knee tendon and ligament by using 3D ultrashort echo time cones (UTE cones) magnetc resonance imaging. Biomed Res Int 2019:8597423. 